\title{
Consultores Ad hoc - 2018
}

Para atender à demanda de avaliação dos artigos submetidos ao BOLEMA temos contado com os membros do Conselho Editorial ${ }^{1}$ e com colaboradores ad hoc. Durante o ano de 2018, colaboraram com o BOLEMA, como pareceristas ad hoc, os seguintes pesquisadores:

Adlai Ralph Detoni (UFJF-MG), Adriana Richit (UFFS-RS), Adriano Vargas (UFF-RJ), Afonso Henriques (UESC-BA), Airton Carrião Machado (UFMG-MG), Albert Mallart (Universidad de Barcelona - Espanha), Aldo Parra (Centro Indigena de Investigaciones Interculturales de Tierradentro - Colômbia), Alessandro Jaques Ribeiro (UFABC-SP), Alexander Maz-Machado (Universidad de Cordoba - Espanha), Amanda Amantes Neiva Ribeiro (UFBA-BA), Ana Leticia Losano (UNICAMP-SP), Ana Cristina Ferreira (UFOPMG), Ana Caballero (Universidad de Extremadura - Espanha), Andriceli Richit (IFSC-SC), Andrew Brantlinger (University of Maryland - EUA), Ángel Alsina (Universitat de Girona Espanha), Ängel Gutiérrez (Universidade de Valência - Espanha), Angela Marta Pereira das Dores Savioli (UEL-PR), Assumpta Estrada (Universitat de Lleida - Espanha), Agustín Grijalva (Universidad de Sonora - México), Beatriz Salleme Corrêa Cortela (UNESP-SP), Belén Giacomone (Universidad de Granada - Espanha), Berta Barquero (Universidad de Barcelona - Espanha), Carles Dorce (Universitat de Barcelona - Espanha), Carlos Rondero (Universidad Autonoma del Estado de Hidalgo - México), Carmen Batanero (Universidad de Granada - Espanha), Carmen Ordóñez (Universidad de Jaén - Espanha), Celil Ekici (Texas A\&M University - EUA), Celina Aparecida Almeida Pereira Abar (PUC-SP), Claudia Vásquez Ortiz (PUC - Chile), Claudinei Santana (UESB-BA), Claudio Fuentealba (Universidad Austral de Chile - Chile), Chris Rasmussen (San Diego State University - EUA), Dario Fiorentini (UNICAMP-SP), Deise Aparecida Peralta (UNESP-SP), Deise Lago Pereira Souto (UNEMAT-MT), Denizalde Pereira (UNEMAT-MT), Dinazar Escudero-Avila (Benemerita Universidad Autonoma de Puebla - México), Duelci Aparecido de Freitas Vaz (PUC-GO), Eliana Alves Pereira Leite (UNIR-RO), Eliane Maria de Oliveira Araman (UTFPR-PR), Elisangela Pavanelo (UNESP-SP), Enrique Mateus Nieves (Universidad Externado de Colômbia - Colômbia), Ernani Martins dos Santos (UPE-PE), Eugenio Lizarde (Escuela Normal Rural Gral. Matías Ramos Santos - México), Fabian Arley Posada-Balvin (UFRN-RN), Fabiane Cristina Höpner Noguti (UFSM-RS), Fabiane Mondini (UNESP-SP), Farhad Kazemi (Islamic Azad University - Irã), Fernanda Malinosky Coelho da Rosa (UFMSMS), Fernando Hitt (UQAM Faculte des Sciences - Canada), Flávia Coura (UFSJ-MG), Fredy Gonzalez (UFRN-RN), Gemma Sala (Universitat de Barcelona Facultat d'Educació Espanha), Gerson Pastre de Oliveira (PUC-SP), Gloria Inés Neira Sanabria (Universidad Distrital Francisco José Caldas - Colômbia), Gunhan Caglayan (New Jersey City University EUA), Gustavo Martínez-Sierra (Universidad Autonoma de Guerrero - México), Hanna Nadim Haydar (Brooklyn College - EUA), Helena Rocha (Universidade Nova de Lisboa - Portugal), Helber Almeida (UFCG-PB), Inés María Gómez-Chacón (Universidad Complutense de Madrid - Espanha), Inocêncio Fernandes Balieiro Filho (UNESP-SP), Ismenia Guzmán

\footnotetext{
${ }^{1}$ Os membros do Conselho Editorial estão disponíveis em: http://www.scielo.br/revistas/bolema/pedboard.htm.
} 
(Universidad de Los Lagos - Chile), Irene Cazorla (UESC-BA), Janeth Amparo Cárdenas (Universidad de Extremadura - Espanha), Jean-Claude Régnier (Université de Lyon - França), Jhony Alexander Villa-Ochoa (Universidad de Antioquia - Colômbia), João Alberto da Silva (FURG-RS), João Bosco Laudares (PUC-MG), José Armando Valente (UNICAMP-SP), José Fernandes da Silva (IFMG-MG), José Luiz Freitas (UFMS-MS), José Vilani Farias (IFRNRN), Jordi Deulofeu Piquet (Universitat Autònoma de Barcelona - Espanha), (Juan Godino (Universidad de Granada - Espanha), Jussara de Loiola Araújo (UFMG-MG), Karla Aparecida Lovis (IFPR-PR), Keith Jone (University of Southampton - Reino Unido), Keith Weber (Rutgers The State University of New Jersey - EUA), Keli Cristina Conti (UFMG-MG), Klinger Teodoro Ciríaco (UFSCAR-SP), Lawrence M. Clark (University of Maryland - EUA), Lidia Hernandez (Benemerita Universidad Autonoma de Puebla - México), Lourdes Ordóñez (Universidad de Jaen - Espanha), Lucas Carato Mazzi (UNESP-SP), Luciane de Fatima Bertini (UNIFESP-SP), Luis Roberto Pino-Fan (Universidad de Los Lagos - Chile), Luiz Marcio Santos Faria (UFBA-BA), Manoel Oriosvaldo Moura (USP-SP), Manuela David (UFMGMG), Marcela Leite (UFTPR-PR), Marcelo Bezerra de Morais (UERN-RN), Márcia Fusaro (UFRJ-RJ), Marcel Puchulu (Universidad Nacional de Villa Maria - Argentina), Marcelo Almeida Bairral (UFRRJ-RJ), Marcio Urel Rodrigues (UNEMAT-MT), Marco Antônio Escher (UFJF-MG), Maria Alexandra Mariotti (Università degli studi di Siena - Itália), Maria do Carmo de Sousa (UFSCAR-SP), Maria Célia Leme da Silva (UNIFESP-SP), Maria Cristina Ferreira (UFMG-MG), Maria Cristina Oliveira (UFJF-MG), María José Seckel (Universidad Católica del Maule - Chile), María Laura Distefano (Universidad Nacional de Mar del Plata Argentina), Maria Lucia Panossiam (UTFPR-PR), María Luz Callejo (Universidad de Alicante - Espanha), Maria Madalena Dullius (Univates-RS), María M. Gea (Universidad de Granada - Espanha), Maria Rosa Massa (Universitat Autònoma de Barcelona - Espanha), Maria Tereza Zampieri (UNESP-SP), Marilena Bittar (UFMS-MS), Martín Acosta (Universidad Distrital Francisco Jose de Caldas - Colômbia), Michele Dias Veronez (UNESPAR-PR), Miguel Montes (Universidad de Huelva - Espanha), Miguel Picado Universidad Nacional de Costa Rica - Casto Rica), Miguel Ribeiro (UNICAMP-SP), Miguel Rodríguez Wilhelmi (Universidad Publica de Navarra - Espanha), Nelson Pirola (UNESP-SP), Nilce Fátima Scheffer (UFFS-SC), Olive Chapman (University of Calgary - Canada), Orlando Figueiredo (UNESP-SP), Oscar Javier Molina (Universidad Pedagogica Nacional - Colômbia), Pablo Beltrán-Pellicer (Universidad de Zaragoza Facultad de Educacion - Espanha), Paola Valero (Universidad de Aalborg - Dinamarca), Patrick Scott (New México State University - EUA), Pedro Javier Rojas (Universidad Distrital Francisco Jose de Caldas - Colômbia), Publio Suarez (Universidad Pedagogica y Tecnologica de Colômbia - Colômbia), Raquel Factori Canova (Universidade Anhanguera-SP), Rejane Waiandt Schuwartz de Carvalho Faria (UFPA-PA), Rodolfo Vergel (Universidad Distrital Francisco Jose de Caldas - Colômbia), Rosa María Farfán Márquez (Centro de Investigación y de Estudios Avanzados del Instituto Politecnico Nacional - México), Rosilda dos Santos Morais (UNIFESP-SP), Rute Borba (UFPE-PE), Samuel Edmundo Lopez Bello (UFRGS-RS), Sandra Malta Barbosa (UEL-PR), Sani Carvalho Rutz da Silva (UTFPR-PR), Santiago Inzunza (Universidad Autónoma de Sinaloa México), Silvana Santos (UFV-MG), Sérgio Carrazedo Dantas (UNESPAR-PR), Silvia Ibarra (Universidad de Sonora - México), Silvia Machado (PUC-SP), Silvia Regina Vieira da Silva (UNESP-SP), Tânia Cristina Rocha Silva Gusmão (UESB-BA), Teresa F. Blanco (Universidade de Santiago de Compostela Facultade de Ciencias da Educacion - Espanha), Teresinha Kawasaki (UFMG-MG), Tiago Kluber (UNIOESTE-PR), Uldarico Malaspina Jurado (Pontificia Universidad Católica del Perú - Peru), Vanessa Cerignoni Benites Bonetti (UNESP-SP), Vanessa Moretti (UNIFESP-SP), Vanessa Oechsler (IFSC-SC), Vera Merlini (UESC-BA), Veridiana Rezende (UNESPAR-PR), Victor Giraldo (UFRJ-RJ), Vinicius Pazuch (UFABC-SP), Wellington Lima Cedro (UFG-GO), Walter Fernando Castro 
(Universidad de Antioquia - Colômbia), Wilson Gordillo (Universidad Distrital Francisco José de Caldas - Colômbia), Yuly Marsela Vanegas (Universitat de Barcelona - Espanha). 